\title{
Phosphate transfer in activated protein complexes expose interaction sites
}

\author{
Sem Tamara, ${ }^{[a]}$ Richard A. Scheltema,${ }^{[a]}$ Albert J. R. Heck, ${ }^{\text {[a] }}$ Aneika C. Leney ${ }^{*[a]}$
}

1 Abstract: For many proteins phosphorylation regulates the 39

2 interaction with other biomolecules. Here, we describe an unexpecte 40

3 phenomenon whereby within a binding interface phosphate group $\$$

4 transfer non-enzymatically from one interaction partner to the oth 42

5 upon activation in the gas-phase. Providing that a high affinity exis $\$ 3$

6 between the donor and acceptor sites, this phosphate transfer is ve 4

7 efficient and the phosphate groups only ligate to sites in proximity 5

8 the binding region. Consequently, such phosphate transfer reaction $\$ 6$

9 may define with high precision the binding site between 47

10 phosphoprotein and its binding partner, and in addition reveal that th 48

11 binding site in this system is retained in the phase transfer fro 49 solution to the gas-phase.

The transfer of a phosphate group to a protein is a ket/3 regulator in protein function ${ }^{[1,2]}$. Phosphorylation can be a striet 4 pre-requisite for protein interactions. This is exemplified $\mathrm{b} / 5$ proteins containing $\mathrm{SH} 2$ domains. In these, the $\mathrm{SH} 2$ domain $\$ 56$ crucial for interactions with phosphorylated tyrosine residues7 gulating signaling in receptor tyrosine kinase pathways 3.8 phosphod phosphorylated the substrate interacts with the ligase, becomes 1 ubiquitinated, and is targeted to the proteasome for degradation ${ }^{4} 62$ Although the frequency of phosphorylation-mediated interaction $\$ 3$ in cells is high, fundamental knowledge is often lacking as to hoß 84 phosphorylation governs this interaction.

One, less characterized interaction, is that between the 6 peptidyl-prolyl cis-trans isomerase (PPlase), Pin1 and it $\$ 7$ phosphoprotein substrates. Pin1 comprises two domains; a 158 terminal WW domain and a C-terminal PPlase domain ${ }^{[5]}$. The9 presence of a WW domain in Pin1, a protein module whict 0 facilitates binding to phosphorylated motifs, makes it unique 1 within the PPlase family ${ }^{[6]}$. Thus, Pin1 catalyzes the cis-tran\$2 isomerization of specifically phosphorylated Ser/Thr-Pro bonds.3 This isomerization in turn can regulate protein dephosphorylation 4 since many phosphatases only act on substrates comprising 95 specific prolyl peptide bond conformation ${ }^{[7]}$. Pin1 is involved in the 6 regulation of transcription and pre-mRNA processing ${ }^{[8]}$. Here,7 Pin1 binds to the phosphorylated C-terminus of RNA polymerase 8

[a] S. Tamara, Dr. R. A. Scheltema, Prof. Dr. A. J. R. Heck, Dr. A. C. Leney

Biomolecular Mass Spectrometry and Proteomics

Bijvoet Center for Biomolecular Research and Utrecht Institute for Pharmaceutical Sciences and Netherlands Proteomics Centre Utrecht University, Padualaan 8, $3584 \mathrm{CH}$, Utrecht (The Netherlands)

E-mail: a.j.r.heck@uu.nl, a.c.leney@uu.nl
(RNAP CTD), modulating its phosphorylation status and thus its ability to transcribe genes ${ }^{[9]}$. Pin1 also regulates transcription in response to TGF-beta signaling through its interaction with phosphorylated Smad3 ${ }^{[10,11]}$. Recently, Pin1 dysfunction has also been linked to Alzheimer's disease progression ${ }^{[12]}$ and Asthma ${ }^{[13]}$. Therefore, it is critical to investigate the mechanism by which Pin1, in a phosphorylation-dependent manner, binds its interaction partners.

Methods are needed to monitor when and under which conditions phosphorylation-dependent interactions occur. Ideally, these methods would monitor complex formation, the number of phosphorylation sites required for binding, and localize precisely the phosphorylation sites of interest ${ }^{[14]}$. Native MS, a technique whereby biomolecules are analyzed in their non-denatured state ${ }^{[15]}$, provides this insight into phosphorylation-dependent non-covalent protein complex formation ${ }^{[14,16]}$. In combination with tandem MS, native MS can locate phosphorylation sites and reveal the stoichiometry of proteins within large macromolecular complexes and their interaction networks ${ }^{[17]}$. These experiments, however, rely on the products of dissociation in the gas-phase accurately reflecting the assembly partners in solution. Indeed, this has proven highly successful in the analysis of many large protein complexes such as V-type ATPases ${ }^{[18]}$, ribosomes ${ }^{[19]}$ and the 195 proteosome ${ }^{[20]}$.

Here, in an investigation focusing on Pin1-phosphopeptide complexes, we stumbled upon an unanticipated phenomenon, whereby the products of gas-phase dissociation no longer reflected the original constituents in solution. Instead, phosphate moieties moved from the original phosphopeptides to proximate acceptor sites on Pin1, providing information on the location of the binding site. We first observed this phenomenon in experiments whereby Pin1 was incubated with a doubly phosphorylated peptide mimicking its known protein binding partner, termed RNAP CTD (Table S1). As expected, based on earlier data ${ }^{[21,22]}$, we observed a 1:1 Pin1:RNAP CTD complex (Figure 1, Figure $\mathrm{S} 1 \mathrm{~A}, \mathrm{D})$. The affinity estimated by native MS $(\mathrm{Kd}=29 \pm 12 \mu \mathrm{M})$ is consistent with the reported affinity of RNAP CTD to the WW domain of Pin1 ${ }^{[22]}$. Next, the Pin1-RNAP CTD complex was subjected to collisional induced dissociation (CID). We expected that in these CID experiments the non-covalent interaction would break resulting in the complex dissociating into its original constituents, i.e. Pin1 and the doubly phosphorylated RNAP CTD. However, the most dominant peaks observed correspond to Pin1 with one or two phosphates covalently bound suggesting that phosphate groups have been transferred from RNAP CTD to Pin1 (Figure 1, Figure S2A). In line with this observation, we also observed the complementary fragment ions corresponding to the singly phosphorylated and unphosphorylated RNAP CTD (Figure 1, Figure S2A). The formation of phosphorylated Pin1 correlates well with the dephosphorylation of RNAP CTD, when monitored as a function of CID energy (Figure S3A). Thus, phosphate 

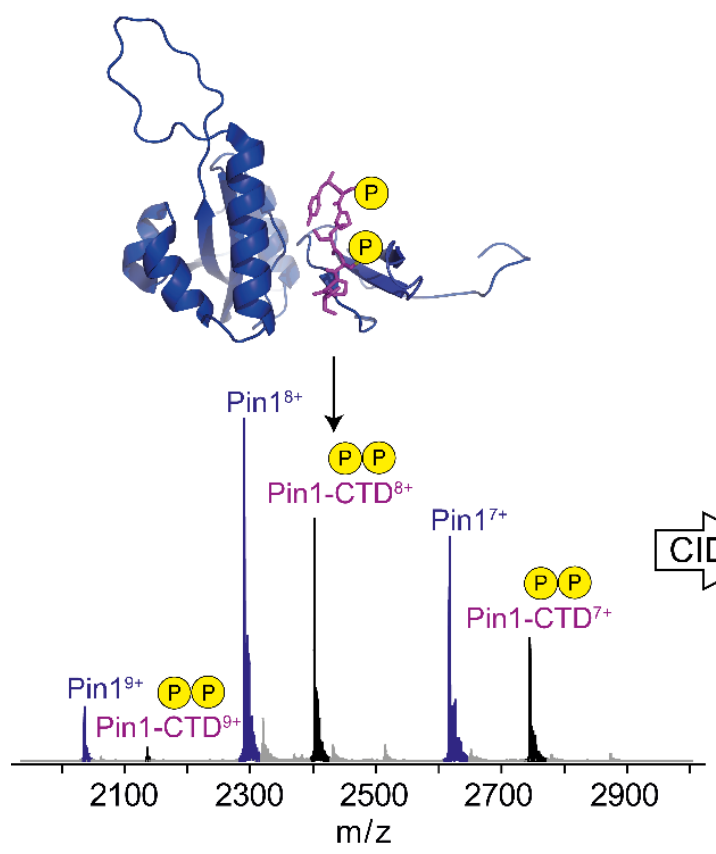
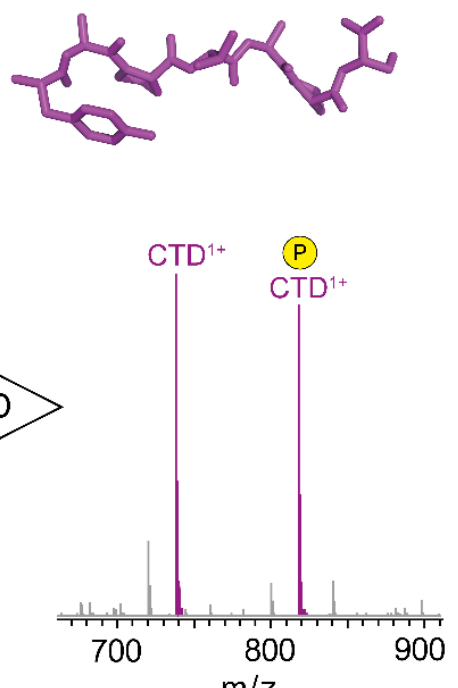

$\mathrm{m} / \mathrm{z}$
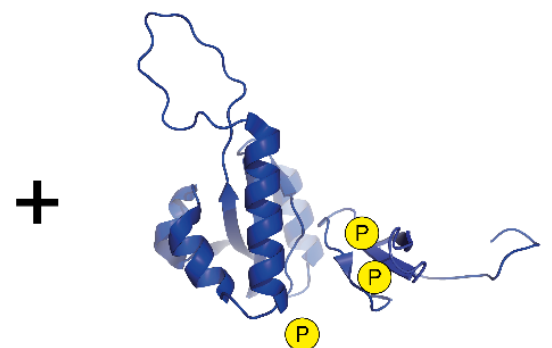

(P)

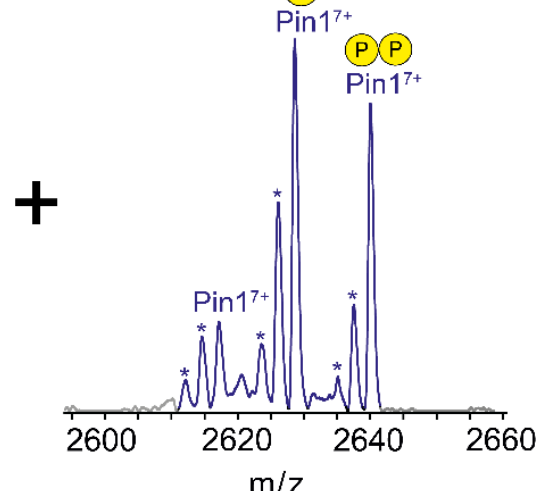

Figure 1. Native (tandem) MS spectra of the Pin1-RNAP CTD (CTD) complex (left) and its dissociation products (right). Phosphate moieties transfer from phosphorylated RNAP-CTD to Pin1 resulting in the formation of doubly- and singly-phosphorylated Pin1 and non-phosphorylated RNAP CTD.

transfer fully precludes dissociation upon Pin1-RNAP CTB2 activation (Figure 1).

Intrigued by our observations, we sought whether this 4 phenomenon was peptide independent. Thus, another5 phosphorylated peptide was chosen corresponding to residue 36 202-215 of Smad3 (Table S1) and incubated with Pin B.7 Comparable to data for the Pin1-RNAP CTD complex, a 1:1 Pin $3-8$ Smad3 complex was observed with an estimated somewhat 9 smaller dissociation constant of $57 \pm 20 \mu \mathrm{M}$ suggesting Smad3 450 also bound to the WW domain of Pin1 (Figure S1B, D). LikewisA,1 upon CID, phosphate transfer between phosphorylated Smad $\$ 2$ and Pin1 was observed, albeit to a lesser extent (Figure S2BA,3 S3B).

To eliminate the possibility that the phosphate transfer hatt5 occurred already in solution prior to gas-phase analysis, the Pin 46 RNAP CTD complex was formed in solution and subsequent 47 dissociated prior to MS analysis (Figure S2D). In thest8 experiments, no phosphorylation was detected on Pin1. Thus, w9 conclude that phosphate transfer exclusively occurs upon gas 50 phase activation of the Pin1-phosphopeptide complex and not $\mathbf{5 1}$ solution.

We hypothesized that the extent of phosphate transfer upo5n CID (Figure S2) might correlate with binding affinity, thu\$4 occurring predominantly within high affinity, specific Pin D-5 phosphopeptide complexes. To test this, RNAP CTD wa\$6 incubated with cytochrome $C$ (CytC); a protein with an alike ML 57 as Pin1, but with no known specific interaction with RNAP CTD.8 Weak ion signals corresponding to a low abundant CytC:RNA $\overline{9} 9$ CTD complex were detected, likely formed by non-speciffo 0 interactions that can occur during the ESI process ${ }^{[23,24]}$ (Figu@1 S1C, D). Consistent with our hypothesis, upon CID of this lov 2 affinity non-covalent complex no phosphate transfer was observed between CytC and RNAP CTD. Instead, we observed the predicted formation of CytC and phosphorylated RNAP CTD as product ions (Figure S2C, S3C). Thus, we conclude that phosphate transfer is likely specific to protein-phosphopeptide complexes whereby the phosphopeptide is bound tightly within the binding site. Consistent with our data, phosphate transfer has previously been observed in the gas-phase within phosphopeptides and during the dissociation of phosphopeptide dimers $^{[25]}$. However, to our knowledge, this is the first instance of phosphate transfer within a non-covalent protein complex, our findings having significant implications on the analysis of structural biology-based MS experiments on phosphoproteinprotein complexes.

In the reported crystal structure of the Pin1-RNAP CTD complex, the phosphoserine at Ser5 on RNAP CTD interacts strongly to Pin1 through hydrogen bonding to Ser16, Arg17 and Tyr23 ${ }^{[22]}$. If such a structure would be largely retained in the gasphase, we would expect the phosphate group on Ser5 to most likely migrate to one of these aforementioned Pin1 acceptor residues. To validate this hypothesis, further top-down fragmentation (i.e. MS3) was performed on the doubly phosphorylated Pin1 fragment ions (Figure S4A). Fragments were observed throughout the entire Pin1 sequence enabling us to accurately pin-point the phosphorylation sites within the WW domain. Short singly-phosphorylated fragments exclusive for the WW domain together with long doubly-phosphorylated fragments spanning across both domains locate the phosphorylation sites in Pin1 to residues in between 16-23 (Figure S4B). Upon comparison of all possible transfer sites within the Pin1 sequence, we found that all these possible phosphosites on Pin1 are within 
$110 \AA$ of pSer5 in the crystal structure. Interestingly, Ser16, Arg 138 2 and Tyr23 in Pin1 are the closest residues to pSer5 an 39 3 consistently display in our data a high number of characteristic 4 phosphorylated fragments (Figure 2), thus these are the mosts 5 likely transfer sites on Pin1. For the transfer of the seconष5 6 phosphate (pSer2), it is likely that the phosphate migrates $\$ 4$ 7 multiple sites within the binding region since this side chain is 8 more flexible and less stabilized in the crystal structure. 9

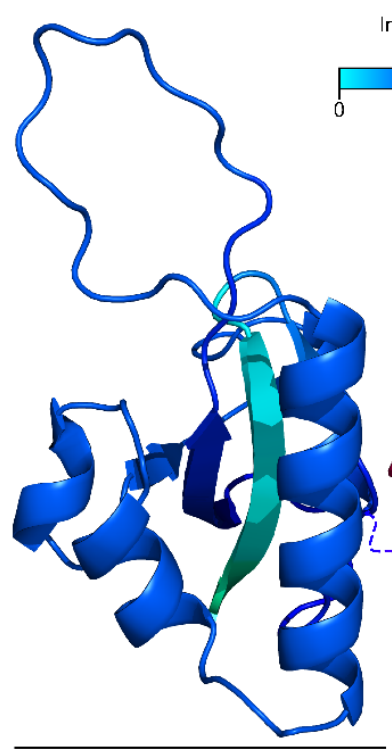

PPlase domain
Intensity-weighted number of phosphorylated fragments
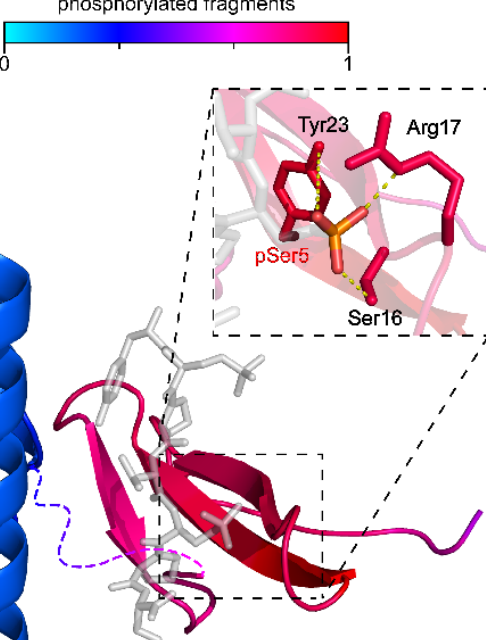

\section{Acknowledgements}

6 We thank Dr. G. E. Folkers for assistance with Pin1 expression 8 Initiative Proteins@Work (project number 184.032.201), the 9 Netherlands Organization for Scientific Research (NWO) and 0 through the European Union Horizon 2020 programme FET1 OPEN project MSmed, Project 686547.

2 Keywords: top-down proteomics, Pin1, RNA polymerase CTD, Figure 2. Crystal structure of Pin1-RNAP CTD (PDB entry: 1f8a) color codeg corresponding to the intensity-weighted number of observed phosphorylatec fragments. RNAP CTD is shown in grey. The interaction of pSer5 (CTD) wito Tyr23, Arg17, and Ser16 (Pin1) is displayed in the inset. The dotted line corresponds to residues absent in the crystal structure.

In summary, we show that phosphate groups within a nornf covalent complex can transfer from one to the other binding 3 partner upon gas-phase activation. This phosphate transferf occurs in tightly interacting complexes whereby the phosphate 6 9 group is located at the binding interface and crucial for the interaction. Since phosphate transfer only occurs to phosphate receptor residues in close proximity, location of these phosphat receptor sites in combination with the location of the origin phosphosite can together provide valuable information on protei 83 protein interaction interfaces. We anticipate that this finding is n\&4 unique to Pin 1 and could have broader implications in the conte 86 of other high affinity phosphorylation-dependent biomolecularf interactions.

\section{Experimental Section}

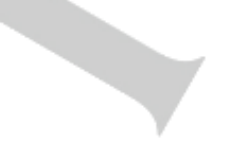
For complex formation, Pin1/CytC was incubated with a 5-fold excess 696 either a phosphopeptide mimicking the C-terminal domain of RNG polymerase or SMAD3. Binding affinities were calculated at different ligang 8 concentrations and non-specific binding corrected for using the reference protein method ${ }^{[23]}$. Mass spectra were acquired by direct infusion using 8 (6) nanoESI source coupled to either and Orbitrap EMR or Orbitrap Fus

35 Lumos mass spectrometer. To monitor the phosphate transfer reaction 6,3

36 the most abundant charge state $\left(8^{+}\right)$corresponding to the PipO4 7 and purification. This work was supported by the Roadmap 3 phosphate transfer, native MS

collisional induced dissociation (a type of CID specific to the Orbitrap using a normalized collision energy of 5-30. For phosphate fragment ions formed following CID were mass selected and further subjected to $E T h c D$ fragmentation. All Pin1 fragments were assigned using an in-house developed data analysis software ${ }^{[16]}$. More details are available in the Supporting Information.

P. Cohen, Trends Biochem. Sci. 2000, 25, 596-601. J. Ptacek, M. Snyder, Trends Genet. 2006, 22, 545-554. J. Schlessinger, Curr. Opin. Genet. Dev. 1994, 4, 25-30. L. J. Holt, FEBS Lett. 2012, 586, 2773-2777.

K. P. Lu, X. Z. Zhou, Nat. Rev. Mol. Cell Biol. 2007, 8, 904-916. Y.-C. Liou, X. Z. Zhou, K. P. Lu, Trends Biochem. Sci. 2011, 36 , 501-514.

J. E. Mayfield, S. Fan, S. Wei, M. Zhang, B. Li, A. D. Ellington, F. A. Etzkorn, Y. J. Zhang, ACS Chem. Biol. 2015, 10, 2405-2414. Y.-X. Xu, Y. Hirose, X. Z. Zhou, K. P. Lu, J. L. Manley, Genes Dev. 2003, 17, 2765-2776.

[9] Y.-X. Xu, J. L. Manley, Genes Dev. 2007, 21, 2950-2962.

[10] A. Nakano, D. Koinuma, K. Miyazawa, T. Uchida, M. Saitoh, M. Kawabata, J. Hanai, H. Akiyama, M. Abe, K. Miyazono, et al., J. Biol. Chem. 2009, 284, 6109-6115.

[11] I. Matsuura, K.-N. Chiang, C.-Y. Lai, D. He, G. Wang, R. Ramkumar, T. Uchida, A. Ryo, K. Lu, F. Liu, J. Biol. Chem. 2010, 285, 1754-1764.

[12] D. A. Butterfield, H. M. Abdul, W. Opii, S. F. Newman, G. Joshi, M. A. Ansari, R. Sultana, J. Neurochem. 2006, 98, 1697-1706. Z.-J. Shen, S. Esnault, A. Schinzel, C. Borner, J. S. Malter, Nat. Immunol. 2009, 10, 257-265.

M. van de Waterbeemd, P. Lössl, V. Gautier, F. Marino, M. Yamashita, E. Conti, A. Scholten, A. J. R. Heck, Angew. Chemie Int. Ed. 2014, 53, 9660-9664

[15] A. C. Leney, A. J. R. Heck, J. Am. Soc. Mass Spectrom. 2017, 28, 5-13.

[16] P. Lössl, A. M. Brunner, F. Liu, A. C. Leney, M. Yamashita, R. A. Scheltema, A. J. R. Heck, ACS Cent Sci. 2016, 2, 445-455.

[17] H. Hernández, C. V. Robinson, Nat. Protoc. 2007, 2, 715-726. Matak-Vinkovic, T. Murata, R. A. Bernal, D. Stock, C. V. Robinson, Science (80-. ). 2011, 334, 380-385.

[19] M. van de Waterbeemd, K. L. Fort, D. Boll, M. Reinhardt-Szyba, A. Routh, A. Makarov, A. J. R. Heck, Nat. Methods 2017, 14, 283-286. A. Politis, F. Stengel, Z. Hall, H. Hernández, A. Leitner, T.

Walzthoeni, C. V. Robinson, R. Aebersold, Nat. Methods 2014, 11, 403-406.

[21] J. P. O'Brien, W. Li, Y. Zhang, J. S. Brodbelt, J. Am. Chem. Soc. 2014, 136, 12920-8.

[22] M. A. Verdecia, M. E. Bowman, K. P. Lu, T. Hunter, J. P. Noel, Nat. Struct. Mol. Biol. 2000, 7, 639-643.

[23] J. Sun, E. N. Kitova, W. Wang, J. S. Klassen, Anal. Chem. 2006, 78, 3010-3018.

[24] E. N. Kitova, A. El-Hawiet, P. D. Schnier, J. S. Klassen, J. Am. Soc. Mass Spectrom. 2012, 23, 431-441.

[25] M.-B. Gonzalez-Sanchez, F. Lanucara, G. E. Hardman, C. E. Eyers, Int. J. Mass Spectrom. 2014, 367, 28-34. 


\section{COMMUNICATION}

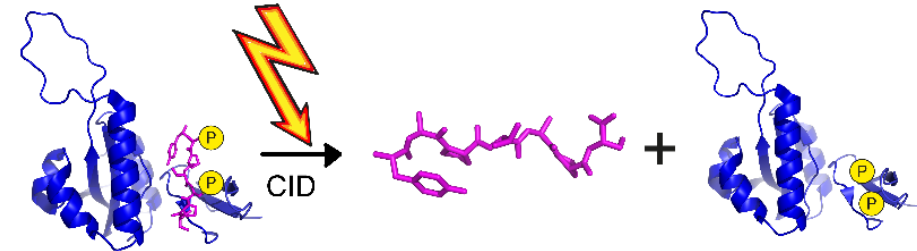

Phosphate groups transfer non-enzymatically from one interaction partner to another during gas-phase activation. In high affinity complexes, this phosphate transfers within the binding site revealing the interaction interface between the proteinphosphopeptide complex.
Sem Tamara, Richard A. Scheltema, Albert J.R. Heck*, Aneika C. Leney*

Page No. - Page No.

Phosphate transfer in activated protein complexes expose interaction sites 